\title{
The P4SR index-a critique of a recent criticism
}

\author{
R. K. MACPHERSON \\ The Department of Environmental Health, School of Public Health and Tropical Medicine, \\ University of Sydney, Sydney, Australia
}

In the October 1967 issue of this Journal there appeared a paper, entitled 'Assessing the Heat Stress and Establishing the Limits for Work in a Hot Mine' (Wyndham, Allan, Bredell, and Andrew, 1967), in which an account was given of an investigation conducted underground at Mount Isa Mine in Queensland. The purpose of the investigation was twofold: to determine which of three commonly used indices of heat stress - wet-bulb temperature, corrected effective temperature, or the P4SR index predicts most accurately the effects on workmen underground of the several factors concerned in heat stress, and to decide, in terms of the selected index, at what level of heat stress the length of shift should be reduced or work should cease.

The reason for undertaking the enquiry, despite the fact that heat-work tolerance has been investigated many times before, is stated by Professor Wyndham and his co-authors (p. $256,{ }^{1}$ col. 1) to be that:

‘. Nearly all the conclusions are based upon experiments in hot rooms on subjects who were artificially acclimatized to heat and who are, in general, quite different from the ordinary workmen in hot industries with respect to age, physical fitness, health, nutrition, and state of acclimatization. It is doubtful, therefore, whether the conclusions based upon hot room studies can be applied in the practical work situation.'

In order to avoid these difficulties a field survey was carried out in which the oral temperatures of 86 miners were taken underground at the end of the third hour of their shift and, at the same time, the dry-bulb, wet-bulb, and globe-thermometer temperatures and the air speed were measured at the place of work. So that the results of these

${ }^{1}$ Page and figure numbers, unqualified, refer to the paper in question. observations 'on the job' could be compared with those of a laboratory type experiment, a subsidiary, more formal investigation was also conducted in which measurements of body temperature, heart rate, and weight loss were made on 29 volunteers engaged in step-climbing in a variety of environmental conditions. However, in the event it happened that the men who volunteered for the subsidiary experiment were both younger and fitter than the general population of miners. Nevertheless, the behaviour of the two groups proved to be so alike (p. 261, col. 1 and col. 2) that it was found possible to combine the two sets of results, and the authors later (p. 264, col. 1 and col. 2, and Fig. 6) showed that the degree of acclimatization possessed by the combined groups was equal to that of the highly acclimatized subjects used in laboratory experiments ('hot room studies') in London and in Singapore (Macpherson, 1960).

The issues of age, physical fitness, and acclimatization having thus been shown to be largely irrelevant, the emphasis of the paper shifts somewhat to establish as its most important experimental finding (p. 255, para. 3) that

$\therefore$. Plots of oral temperature on P4SR for conditions where G.T. was more than $10^{\circ} \mathrm{F}$. above D.B. were found to fall well below the rest of the plots, indicating that P4SR exaggerates the effect of mean radiant temperature.'

and (p. 255, para. 4)

'These results indicate that the emphasis given to G.T. in the P4SR index is too great. . . . It appears, therefore, that the P4SR index should be revised in this regard.'

This is a very disturbing conclusion as the P4SR index is a widely accepted and much used measure of heat stress. Moreover, if the assertion is well founded, it could call into question the validity of 
much work in the field of climatic physiology. The evidence on which it is based, therefore, merits careful examination and that is the chief concern of this paper.

Two arguments are advanced by the authors in support of their conclusions. The first and principal argument (p. 263, col. 2) is that, if the P4SR values for the aberrant 'plots' of oral temperature (that is 'for conditions where G.T. was more than $10^{\circ} \mathrm{F}$. above D.B.') are calculated, neglecting the globethermometer reading entirely, then the points in question fall 'close to the regression line fitted to the rest of the data'. But the regression line already includes the effect of a globe-thermometer increment which is accepted by the investigators (p. 262, col. 1) as correct up to $10^{\circ} \mathrm{F}$. Therefore, the effect of any increment above $10^{\circ} \mathrm{F}$. must have a contrary effect to that below $10^{\circ} \mathrm{F}$. In other words, if the globethermometer increment was $20^{\circ} \mathrm{F}$. then the effect of the increment between $10^{\circ} \mathrm{F}$. and $20^{\circ} \mathrm{F}$. would be equal in magnitude but opposite in sign to the effect from $0^{\circ} \mathrm{F}$. to $10^{\circ} \mathrm{F}$. This is, of course, untenable.

The second, supporting argument is based on a mathematical analysis (Appendix 2), the relevant implications of which are also quoted in the main body of the text (p. 263, col. 2). Of these mathematical findings it may suffice to say that, in addition to the conclusion to which the authors draw attention, viz., that unit increase in the wet-bulb temperature has the greatest effect on the P4SR followed closely by the effect of unit increase in the globe-thermometer temperature, they also indicate that the effect of increasing the dry-bulb temperature is to decrease the value of the P4SR index. No comment is made on this rather surprising conclusion.

Professor Wyndham and his co-authors then state (p. 263, col. 2, and p. 264, col. 1)

'The results of this analysis are at variance with the conclusions reached in the Medical Research Council report issued in February 1954 on this subject (Ferres, Fox, Jack, John, Lind, Macpherscn, and Newling, 1954) which reads:

"Although no absolute value can be assigned to the effects of increasing the mean radiant temperature the experiments of the two series taken together show that, as measured by the amount of sweat loss per kilogram of body weight, increasing the mean radiant temperature $30^{\circ} \mathrm{F}$. above air temperature (as measured by G.T.), at an air temperature of $100^{\circ} \mathrm{F}$., produces approximately the same increase in strain as increasing the air temperature by $10^{\circ} \mathrm{F}$. to $110^{\circ} \mathrm{F} . "$

This statement means that the same effect on P4SR values is seen from a $1^{\circ} \mathrm{F}$. change in D.B. and a $30^{\circ} \mathrm{F}$. change in G.T.'.

It is true that the results of their analysis are at variance with the conclusions of myself and my colleagues, and I have no complaint on that score, but we have been misquoted. The quotation from the paper (Ferres et al., 1954) should read as follows:

'Although no absolute value can be assigned to the effect of increasing the mean radiant temperature, the experiments of Series VI and Series VII taken together show that, as measured by the amount of sweat loss per kilogram of body weight, increasing the mean radiant temperature $30^{\circ} \mathrm{F}$., above air temperature, at an air temperature of $100^{\circ} \mathrm{F}$. produces approximately the same increase in strain as increasing the air temperature by $10^{\circ} \mathrm{F}$. to $110^{\circ} \mathrm{F}$.'

It will be seen that, besides minor alterations of no significance, a phrase '(as measured by G.T.)' has been inserted which does not appear in the original. This serves to make a point in the sentence immediately following in which 'the mean radiant temperature $30^{\circ} \mathrm{F}$. above air temperature' has become 'a $30^{\circ} \mathrm{F}$. change in G.T.'. (This sentence, by the way, contains a misprint - ' $1{ }^{\circ} \mathrm{F}$.' should clearly read ' $10^{\circ} \mathrm{F}$. '.)

This would suggest an unawareness of the difference which exists between the reading of the globe thermometer and the mean radiant temperature of the surroundings which may be deduced from the globe-thermometer reading if the prevailing air temperature and air speed are also known.

Unfortunately, this conclusion forms the basis of a charge of inconsistency on the part of myself and my co-workers. The passage in question (p. 264, col. 1) reads as follows:

'The discrepancy between this conclusion and the weighting given to the G.T. in the calculation of the P4SR values does not seem to have been realized, because in the Medical Research Council's Special Report No. 298 on the Singapore experiments, Macpherson (1960) states:

"It was found that the P4SR nomogram predicts the sweat loss of fit, acclimatized men in hot conditions in which the mean radiant temperature is above air temperature as accurately as it predicts the sweat loss in those environments in which the mean radiant temperature is equal to the air temperature.",

A similar statement also appears in the Appendix to the paper (p. 271, col. 1).

In fact, at the level of radiant heat referred to in the paper by Ferres et al. (1954), a mean radiant temperature of approximately $130^{\circ} \mathrm{F}$. or, more precisely, $132 \cdot 4^{\circ} \mathrm{F}$. the globe-thermometer temperature exceeded the air temperature by an amount, depending on the air speed, ranging from $9.5^{\circ}$ to $11.3^{\circ} \mathrm{F}$., an approximate increment (G.T. - D.B.) of $10^{\circ} \mathrm{F}$. This is indicated in the relevant diagrams. The finding that an increment of $10^{\circ} \mathrm{F}$. in the globethermometer reading is equivalent to a $10^{\circ} \mathrm{F}$. rise in air temperature is not inconsistent with the statement 
that the P4SR predicts sweat losses in conditions in which the mean radiant temperature is above air temperature as accurately as it does when the air temperature is equal to the mean radiant temperature. It is precisely what should happen when allowance is made for the spurious depression of the wet-bulb temperature implied in substituting the globe-thermometer reading for the dry-bulb temperature in the P4SR nomogram.

The next paragraph then states (p. 264, col. 1)

'It appears from this discussion that the method of calculating P4SR values from the nomogram needs to be revised for conditions where the G.T. is higher than D.B. The revision should be in the direction of reducing the present weighting given to the effect of the G.T. Instead of its effect being roughly equal to that of the W.B., as it is at present in the nomogram, it appears that its effect should be only one-third as great as D.B., in the particular range of conditions examined.'

This represents the major scientific finding described in the paper. It is repeated later (p. 267, col. 2) in a slightly different form:

$\therefore$. The results of these studies have shown that the physiological effects of long-wave radiation in the mine at Mount Isa are less than those of either W.B. or D.B. As a rough yardstick it can be taken that the physiological effect of a one-degree Fahrenheit change in G.T. is about one-third the effect of a similar change in D.B. and about one-fifth the effect of a similar change in W.B. in the range of air conditions in the mine at Mount Isa. . '

where it is used as a basis for the establishment of the limits for work in the mine, which was the chief practical outcome of the investigation. However, the claim that the effect of unit change in the globethermometer temperature is quantitatively equivalent to one-third of the effect of unit change in the drybulb temperature is based not on their own findings, with which it is at variance, but apparently on a misunderstanding of the work of others.

Some lesser points deserve comment. The $F$ values quoted on page 260 , columns 1 and 2 , give rise to some concern. Even for 1 and 3 degrees of freedom, $F$ values in excess of 10.13 would be significant at the $5 \%$ level, so that, using standard methods of analysis, $F$ values of 11.71 and 15.02 would indicate that there were highly significant differences in the slopes of the regression lines compared and not that the slopes were without significant differences as stated, although the regression coefficients are, in fact, much the same.

Perhaps the most serious is the apparent inconsistency found on page 262 , column 2 , where it is stated that

'The oral temperatures measured at the end of the third hour of the shift have not been shown to be significantly different from those measured either during the first two hours or in the afternoon after "crib" time.' but, on page 258 , column 2 , it has already been said

‘. From this Table [Table II] it will be seen that the P4SRs decrease steadily over the first three hours of the shift.'

Both these statements can be true only if the P4SR and the oral temperature are not related and the basic assumption in the paper is false. In the experimental protocol quoted (Appendix 1), there is, in the work period shown, a range of oral temperatures from $99 \cdot 0$ to $100 \cdot 6^{\circ} \mathrm{F}$.

Furthermore, in two other places the argument is difficult to follow. The Table, which sets out the increment in wet-bulb temperature necessary to produce unit increases in the P4SR index, is abstracted from the Table in Appendix 2 to the paper (p. 271). The value $34 \cdot 81$ given in all three

\section{TABLE}

INCREMENT IN WeT-Bulb Temperature $\left({ }^{\circ}\right.$ F. $)$ NECESSARY TO PRODUCE AT THREE LEVELS OF Wet-Bulb Temperature and Three Levels of GLOBE-THERMOMETER TEMPERATURE UNIT INCREASE IN THE P4SR INDEX WHEN THE AIR Temperature IS $104^{\circ} \mathrm{F} .1$

\begin{tabular}{|c|c|c|c|}
\hline \multirow{2}{*}{$\begin{array}{c}\text { Level of } \\
\text { wet-bulb } \\
\text { temperature } \\
\left({ }^{\circ} \mathrm{F} .\right)\end{array}$} & \multicolumn{3}{|c|}{ Globe-thermometer temperature $\left({ }^{\circ} \mathrm{F}.\right)$} \\
\hline & 104 & 106 & 108 \\
\hline $\begin{array}{l}80 \\
84 \\
88\end{array}$ & $\begin{array}{r}34 \cdot 81 \\
8.07 \\
4.57\end{array}$ & $\begin{array}{r}34.81 \\
8.07 \\
4.57\end{array}$ & $\begin{array}{r}34 \cdot 81 \\
8 \cdot 07 \\
4 \cdot 57\end{array}$ \\
\hline
\end{tabular}

${ }^{1}$ The air speed is assumed to be $200 \mathrm{ft}$./min. and the metabolic rate $125 \mathrm{kcal} . / \mathrm{m} .{ }^{2} / \mathrm{hr}$.

columns for 'W.B. 80' appears absurd on at least two counts. In the circumstances some comment, or at least a reassurance that this value is indeed the value indicated by their analysis, seems called for, the more especially as a casual check with the P4SR nomogram will show that at G.T. $104^{\circ} \mathrm{F}$., D.B. $104^{\circ}$ F., A.S. $200 \mathrm{ft} . / \mathrm{min}$., M.R. $125 \mathrm{kcal} / \mathrm{m}^{2} / \mathrm{hr}$, increasing the wet-bulb temperature from $80^{\circ} \mathrm{F}$. to $89.8^{\circ} \mathrm{F}$. (an increment of $9.8^{\circ} \mathrm{F}$., not $34.81^{\circ} \mathrm{F}$. as stated) produces unit increase in the P4SR value.

A further example is the assertion (p. 267, col. 1 and col. 2) that, in the special circumstances prevailing underground at Mount Isa, it was found that a mercury thermometer with a blackened bulb gave a reading satisfactorily approximating that of the globe thermometer. This is a surprising finding when it is considered that the convective heat exchange of a body is greatly influenced by its shape and size (Fishenden and Saunders, 1932), a point to which Bedford and Warner (1934) make specific reference 
in their original paper on the use of the globe thermometer. A table setting out the values obtained in the 40 pairs of measurements on which the finding was based would provide much more acceptable substantiation than the unsuitable measure of agreement offered.

It appears that the values given for oral temperature are open to question. For example, a value of $97.8^{\circ} \mathrm{F}$. is given for a P4SR of approximately 5.9 (Figs 1 and 2). It is improbable that even a thermal athlete could be exposed to such a level of work and heat stress for 3 hours and have about noon, when the circadian variation in body temperature is approaching its peak, a true oral temperature below $98^{\circ} \mathrm{F} .{ }^{1}$ As this is one of the crucial values in the experiment, namely one in which the globethermometer temperature exceeded the dry-bulb temperature by more than $10^{\circ} \mathrm{F}$., comment at least is demanded.

It is said (p. 257, col. 1) that 'Work rates of the various tasks were estimated by a physiologist with considerable experience ... but one would ask whether the same experienced physiologist was present on all occasions to make sure that the actual energy expenditure coincided with the estimated expenditure. 'Ordinary shovelling can be regarded as hard work' (p. 257, col. 2) but this, I would suggest, depends on how hard the workman shovels and how often he leans upon his shovel to rest. In short, though useful and entirely suitable in certain circumstances, the method used for estimating energy expenditure is liable to a large experimental error.

The measurement of environmental conditions in a situation such as that described presents considerable difficulty. Globe-thermometer readings refer only to the precise position at which the globe thermometer is situated, and in a highly non-uniform environment large differences can exist in the readings within a distance of a few feet. It would be possible to follow the subject around with a sling psychrometer but not with a globe thermometer which takes 20 minutes to come into equilibrium and, in hot areas especially, the subject might spend only a few minutes in the one place. How this difficulty was overcome is not mentioned in the paper. However, in Appendix 1 (p. 269), the only experimental protocol given, 32 globe-thermometer readings are listed. Of these 32 readings, 11 are indicated to be approximations or estimations. It appears also that, of the 33 occasions on which the

${ }^{1}$ In the P4SR scatter diagrams (Figs 1 and 2) there are four other values below $98^{\circ} \mathrm{F}$. but these are all for P4SR values less than 2. It is noted, however, that there are two still lower values shown at $75^{\circ} \mathrm{F}$. effective temperature and $70^{\circ} \mathrm{F}$. wet-bulb (Fig. 1) which appear to have been omitted from the two P4SR scatter diagrams both of which, nevertheless, show 87 points not 84 , as one would expect if there were, as stated, 86 subjects. wind velocity is recorded in this table, it has been estimated on 23 occasions. The implication is that no great precision was attempted, or indeed intended, in these measurements of mean radiant temperature and air movement.

It may be said that the evidence so far presented is largely circumstantial with respect to the main point at issue. It is, however, substantial and uniformly supports only one conclusion, in the light of which an alternative and more likely explanation of the experimental findings may be advanced.

It is considered that the main piece of direct evidence bearing on the aberrant results is that they are associated not so much with high levels of radiant heat as with high values for the P4SR. Examination of Figs 1 and 2 shows that of the 87 points plotted, apart from those in which the globethermometer increment exceeded $10^{\circ} \mathrm{F}$., there are only two which exceed a P4SR value of 5 .

Indeed it seems that the authors themselves were aware that the aberrant points were associated only with high values for the P4SR, as the following statement (p. 263, col. 2) seems to indicate:

'. . The results of the present study have shown that where G.T. was more than $10^{\circ} \mathrm{F}$. higher than D.B., the P4SR values were high and the oral temperatures of the group of subjects under these conditions fell well below the regression lines fitted to the rest of the data.'

It is therefore suggested that for P4SR values calculated to be 5 or more, if the environmental conditions in the working situation were what they are said to be, then in general, the men exposed to them did not work at the rate necessary for the prescribed P4SR value. We are told (p. 258, col. 1) that '. . . When they feel tired, or uncomfortably hot, they generally withdraw to a cool place to rest', and this is precisely what I think they did at the times in question for the very good reason that they could not, without unacceptable physical distress, do otherwise.

In the subsidiary stepping experiment there were apparently no aberrant values for the oral temperature. It is true that in this experiment high levels of radiant heat were not used (p. 260, col. 2), but the rate of energy expenditure in this case was controlled with some accuracy.

On page 262, column 1, the authors state

'The main conclusion from this analysis is that . . Another is that, because one regression equation can be used for "stepping" and "on the job" oral temperatures against P4SR, the assessment of work rate on the job must be accurate.'

I would submit that a corollary to this argument is - if the oral temperatures 'on the job' do not agree with the regression equation then the assessment of the work rate 'on the job' must be inaccurate and not that the P4SR is in error. 
The remainder of the paper calls for little comment for the quite sufficient reason that much of it including that relating to the practical industrial issue involved, lies outside the scope of this critique. The paper does, however, raise one apropos matter of general interest which merits discussion, namely the comparative reliability of the results of laboratory experiments and of observations made in the field in assigning accurate values to the relative effects of the several factors which determine the level of heat stress.

Professor Wyndham and his co-authors embarked on their investigation underground in the mine because they doubted the applicability of the results of hot-room studies to the practical work situation and, in the interpretation of their results, they clearly hold their observations to be more trustworthy than those made in the laboratory by others. Their claim (p. 256, col. 2) that their studies underground represented a novel approach ('This is the first time, of which we are aware, that any attempt has been made to apply in the practical working places in a mine the methods which have been developed in climatic chambers for assessing heat stress and for establishing the limits of heat stress for work in hot conditions') cannot, of course, be substantiated. The authors themselves refer to the occasion when, more than 60 years ago, Haldane (1905) measured body temperatures, including those of working miners underground in Dolcoath Mine, and from that time onwards the reactions of miners to their working conditions have been studied on innumerable occasions.

It is particularly surprising that Professor Wyndham and his colleagues should ignore the work on the Kolar goldfields of Caplan and Lindsay (1946) in which not only were the oral temperatures, pulse rates, and sweat losses of the subjects measured, but in some cases blood pressure readings were also taken and samples of blood collected for analysis. One point of special importance in this investigation is that, by using hand-drilling as a task and measuring the depth of the hole achieved, an objective measure of the work actually performed was obtained. The observation that as the level of heat stress rose, the energy expended by the miners diminished until a point was reached when little useful work was performed, is of special significance in the present context.

No one would deny the great value of field investigations but it is significant to note how often they are followed by laboratory experiments to confirm or extend the observations made. Haldane, having made his observations in the mine, found it necessary, in order that adequate information might be obtained, to resort to improvised climatic chambers - the incubation room at the Lister Institute and a Turkish bath at Oxford. But perhaps the most convincing evidence of the comparative precision of the measurements made in the two situations is to be found in the conclusions of Bedford and Warner (1931). These two distinguished workers, no strangers to underground workings, give it as their opinion, 'Under actual mining conditions the factors influencing the rate of working are so complex that it is difficult, if not impossible, to obtain data which will enable the relative influences of the dry and wet bulb factors to be accurately assessed. . . . It is concluded that if the relative influences of the dry and wet bulb factors on working capacity are to be accurately assessed, the matter must be investigated under laboratory conditions.' It is my opinion that the paper under discussion amply confirms this conclusion.

\section{References}

Bedford, T., and Warner, C. G. (1931). Observations on the working capacity of coal miners in relation to atmospheric conditions. J. industr. Hyg., 13, 252-260.

- (1934). The globe thermometer in studies of heating and ventilation. J. Hyg. (Lond.), 34, 458-473.

Caplan, A., and Lindsay, J. K. (1946). An experimental investigation of the effects of high temperatures on the efficiency of workers in deep mines. Bull Instn. Min. Metall., No. 480. Also in Trans. Instn. Min. Metall., 1946-47, 56, 165-195.

Ferres, Helen M., Fox, R. H., Jack, J. W., John, R. T., Lind, A. R., Macpherson, R. K., and Newling, P. S. B. (1954). Physiological responses to hot environments of young European men in the tropics. V. The effect of increasing the mean radiant temperature of the surroundings. Med. Res. Coun., Lond., R.N.P. Rep. No. $54 / 812$.

Fishenden, Margaret, and Saunders, O. A. (1932). The Calculation of Heat Transmission. H.M.S.O., London.

Haldane, J. S. (1905). The influence of high air temperatures. No. 1. J. Hyg. (Lond.), 5, 494-513.

Macpherson, R. K. (1960). Physiological Responses to Hot Environments. Med. Res. Coun., Lond., Spec. Rep. Ser. No. 298.

Wyndham, C. H., Allan, A. McD., Bredell, G. A. G., and Andrew, R. (1967). Assessing the heat stress and establishing the limits for work in a hot mine. Brit. J. industr. Med., 24, 255-2 71 .

Received for publication June 17, 1968. 